\title{
X-ray imaging and spectroscopic study of the SNR Kes 73 hosting the magnetar 1E 1841-045
}

\author{
H. S. Kumar ${ }^{1}$, S. Safi-Harb ${ }^{1,2}$, P. O. Slane ${ }^{3}$, and E. V. Gotthelf ${ }^{4}$ \\ ${ }^{1}$ Department of Physics \& Astronomy, University of Manitoba, Winnipeg, Canada \\ email: harsha@physics.umanitoba.ca \\ ${ }^{2}$ Canada Research Chair \\ ${ }^{3}$ Harvard-Smithsonian Center for Astrophysics, 60 Garden Street, Cambridge, MA 02138, USA \\ ${ }^{4}$ Columbia Astrophysics Laboratory, Columbia university, New York, NY 10027, USA
}

\begin{abstract}
We present the first detailed Chandra and XMM-Newton study of the young Galactic supernova remnant (SNR) Kes 73 associated with the anomalous X-ray pulsar (AXP) 1E 1841045. Images of the remnant in the radio $(20 \mathrm{~cm})$, infrared $(24 \mu \mathrm{m})$, and X-rays $(0.5-7 \mathrm{keV})$ reveal a spherical morphology with a bright western limb. High-resolution Chandra images show bright diffuse emission across the remnant, with several small-scale clumpy and knotty structures filling the SNR interior. The overall Chandra and XMM-Newton spectrum of the SNR is best described by a two-component thermal model with the hard component characterized by a low ionization timescale, suggesting that the hot plasma has not yet reached ionization equilibrium. The soft component is characterized by enhanced metal abundances from $\mathrm{Mg}, \mathrm{Si}$, and $\mathrm{S}$, suggesting the presence of metal-rich supernova ejecta. We discuss the explosion properties of the supernova and infer the mass of its progenitor star. Such studies shed light on our understanding of SNRs associated with highly magnetized neutron stars.
\end{abstract}

Keywords. ISM: individual (SNR Kes 73) - pulsars: individual (AXP 1E 1841-045) - supernova remnants - X-rays: ISM

\section{Introduction}

Kes 73 (G27.4+0.0), hosting the anomalous X-ray pulsar (AXP) 1E 1841-045, is a shell-type supernova remnant (SNR) located at a distance of $8.5 \mathrm{kpc}$. At radio wavelengths, the remnant shows a small diameter $\left(\sim 5^{\prime}\right)$ spherical shell characterized by a steep spectral index $(\alpha \sim 0.68)$ and a flux density of $6 \mathrm{Jy}$ at $1 \mathrm{GHz}$ (Kriss et al. 1985). The SNR was clearly detected in the $24 \mu \mathrm{m}$ infrared band with an estimated dust mass of $0.11 M_{\odot}$ (Pinheiro Gonçalves et al. 2011). At X-ray energies, the system reveals the bright magnetar at its center. AXP 1E 1841-045 is the slowest ( $P=11.8 \mathrm{~s})$ known magnetar with a surface dipole magnetic field $B=7.1 \times 10^{14} \mathrm{G}$ and a characteristic age of $\sim 4.7$ kyr (Vasisht \& Gotthelf 1997).

Previous X-ray observations of Kes 73 with $A S C A$ have shown that the SNR emission is dominated by lines from $\mathrm{Mg}$, $\mathrm{Si}$, and $\mathrm{S}$. The $\mathrm{SNR}$ spectrum was best described by a thermal bremsstrahlung model $(k T \sim 0.6 \mathrm{keV})$, with an estimated age of $\leqslant 2000$ years (Gotthelf \& Vasisht 1997). An XMM-Newton study of Kes 73 using only the MOS data argued against the millisecond proto-neutron star model for magnetar formation (Vink \& Kuiper 2006). Another recent study by Lopez et al. (2011) made use of one of the Chandra observations of Kes 73, where the SNR spectrum was fitted by a one-component VPSHOCK model $(k T=0.84 \pm 0.49 \mathrm{keV})$ and reported the detection of enhanced abundances from $\mathrm{Mg}, \mathrm{Si}$, and $\mathrm{S}$. Since all these studies lacked a thorough investigation of Kes 


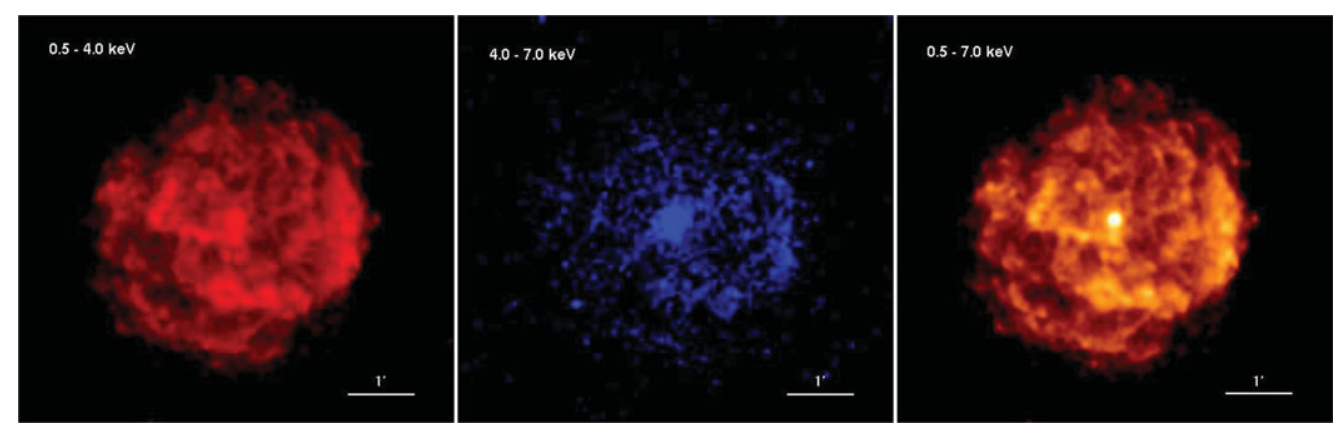

Figure 1. Chandra images of Kes 73 in different energy bands: $0.5-4 \mathrm{keV}$ (left), $4-7 \mathrm{keV}$ (middle), and $0.5-7 \mathrm{keV}$ (right). The images from both Chandra data were exposure-corrected, merged, and smoothed using a Gaussian with $\sigma=1^{\prime \prime}-2^{\prime \prime}$ for a significance of detection 2 to 5 .

73 using all available data, we performed a detailed X-ray imaging and spectroscopic study to address the remnant's multi-wavelength morphology, constrain the SNR spectral parameters and explosion properties, and infer the mass of its progenitor star. We summarize these results briefly below. A detailed analysis and discussion can be found elsewhere (Kumar et al. 2013).

\section{Observations and data analysis}

The SNR Kes 73 was observed with Chandra ACIS on 2000 July 23 (ObsID: 729) and 2006 July 30 (ObsID: 6732). The data were reduced using the standard CIAO routines, yielding a total effective exposure time of $\sim 54 \mathrm{ks}$. XMM-Newton observed Kes 73 on 2002 October 5 and 7 (ObsIDs: 0013340101 and 0013340201) with the European Photon Imaging Camera (EPIC). The data from both the MOS and pn instruments were analyzed using the standard XMMSAS tasks, yielding a total exposure time of $\sim 27 \mathrm{ks}$. X-ray spectra were extracted from various regions across Kes 73 using all datasets, and the background was selected from an adjacent source-free region to the north-east of the remnant. We also extracted the whole SNR region of radius $150^{\prime \prime}$ with the emission from the pulsar $\left(\sim 25^{\prime \prime}\right.$ from XMM-Newton and $\sim 10^{\prime \prime}$ from Chandra) plus other point sources removed. The background was extracted from a ring surrounding the SNR, extending from $150^{\prime \prime}-180^{\prime \prime}$.

\section{Morphology of Kes 73}

In Figure 1, we show the combined Chandra images of Kes 73 in different energy bands: $0.5-4 \mathrm{keV}, 4-7 \mathrm{keV}$, and $0.5-7 \mathrm{keV}$. The remnant has a circular morphology with an apparent diameter of $\leqslant 5^{\prime}$ and reveals small-scale clumpy and bright knotty structures filling the SNR interior. These structures are clearly noticeable in the $0.5-4 \mathrm{keV}$ band, which has an overall morphology similar to that of the broadband $(0.5-7 \mathrm{keV})$ image. The 4-7 keV image shows significant hard X-ray emission originating from the pulsar, in addition to emission from most of the SNR particularly enhanced in the west. An incomplete and outer shell-like feature runs from east to south. The interior region to the east of the AXP shows bright diffuse emission, which extends towards the southern side of the SNR to merge with the bright western limb. The northern portion of the SNR is relatively faint in X-rays, with no distinct shell-like feature.

Figure 2 shows the remnant's $20 \mathrm{~cm}$ radio image obtained with MAGPIS (left) and the $24 \mu \mathrm{m}$ band infrared image obtained with MIPSGAL (right) overlaid on the broadband 

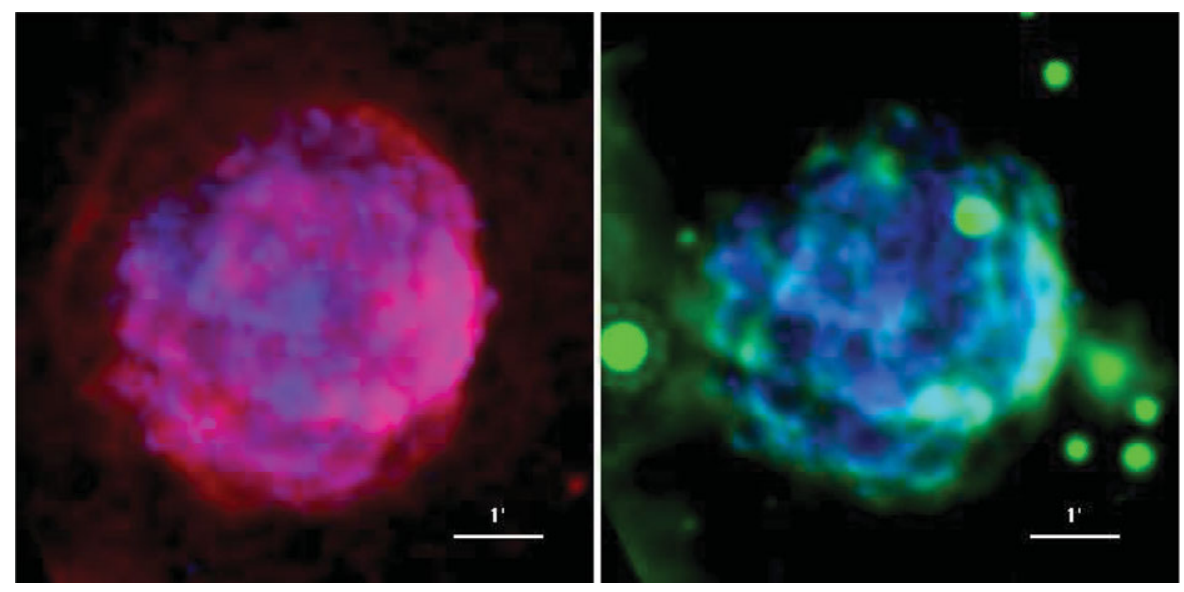

Figure 2. Multi-wavelength morphology of SNR Kes 73. Left: MAGPIS $20 \mathrm{~cm}$ radio image (red) overlaid on the $0.5-7 \mathrm{keV}$ Chandra image (blue). Right: MIPSGAL $24 \mu \mathrm{m}$ band infrared image (green) overlaid on the $0.5-7 \mathrm{keV}$ Chandra image (blue). The radio and IR images have a Gaussian smoothing of $\sigma=3^{\prime \prime}$ and the Chandra image has a Gaussian smoothing of $\sigma=1^{\prime \prime}-2^{\prime \prime}$.

Chandra image. The radio shell is clearly more extended on the north-eastern side of the SNR, displaying a thin filamentary structure along the edges (seen in pure red). This feature is likely the expanding outer blast wave which is not detected in X-rays. The $\mathrm{X}$-ray emission seems to dominate the SNR interior filling the radio shell. The radio shell extending from the eastern limb to the southern edge of the SNR also matches with the corresponding outer shell-like structure in the X-ray image. The Spitzer MIPS image reveals a morphology more comparable to that of the X-ray image with arc-like features and bright infrared emission filling the SNR. The remnant's western limb is very bright in the infrared as well, precisely outlining the radio and X-ray emission. We do not see any significant infrared emission from the north-eastern boundary of the SNR.

\section{Spectral properties and progenitor of Kes 73}

A two-component thermal model best describes the X-ray spectra originating from the small-scale regions (VPSHOCK+VPSHOCK) and the global SNR spectrum (VSEDOV+VPSHOCK; Figure 3). We do not find any significant spatial variations of the spectral parameters across the SNR, except for a slightly elevated column density $N_{H}$ (which ranges between $\sim(2.6-3.3) \times 10^{22} \mathrm{~cm}^{-2}$ from east to west) on the western side with an estimated difference of $\sim 7 \times 10^{21} \mathrm{~cm}^{-2}$ between the eastern and western sides. However, there is no evidence of any cloud or nearby sources interacting with the SNR. The soft components show plasma temperatures $\sim 0.3-0.5 \mathrm{keV}$ and ionization timescales $\gtrsim 10^{12} \mathrm{~cm}^{-3} \mathrm{~s}$ for most of the regions with enhanced metal abundances, while the hard components exhibit plasma temperatures $\sim 1.1-1.7 \mathrm{keV}$ and low ionization timescales $\sim(0.5-2.8) \times 10^{11} \mathrm{~cm}^{-3} \mathrm{~s}$ with solar abundances (except for a slightl y enhanced Fe abundance for some regions). These results indicate that the soft-component arises from the reverse-shocked ejecta with most regions showing a plasma that has reached ionization equilibrium, while the hard-component originates from the shocked interstellar or circumstellar medium. The detection of enhanced Si and S abundances across the remnant suggests that the X-ray emission from the SNR Kes 73 is ejecta-dominated.

Using the global fit to the SNR's spectrum, we infer an SNR age ranging between $\sim 1.1 D_{8.5}$ kyr for the free expansion phase (assuming an expansion velocity of $5000 \mathrm{~km} \mathrm{~s}^{-1}$ ) 


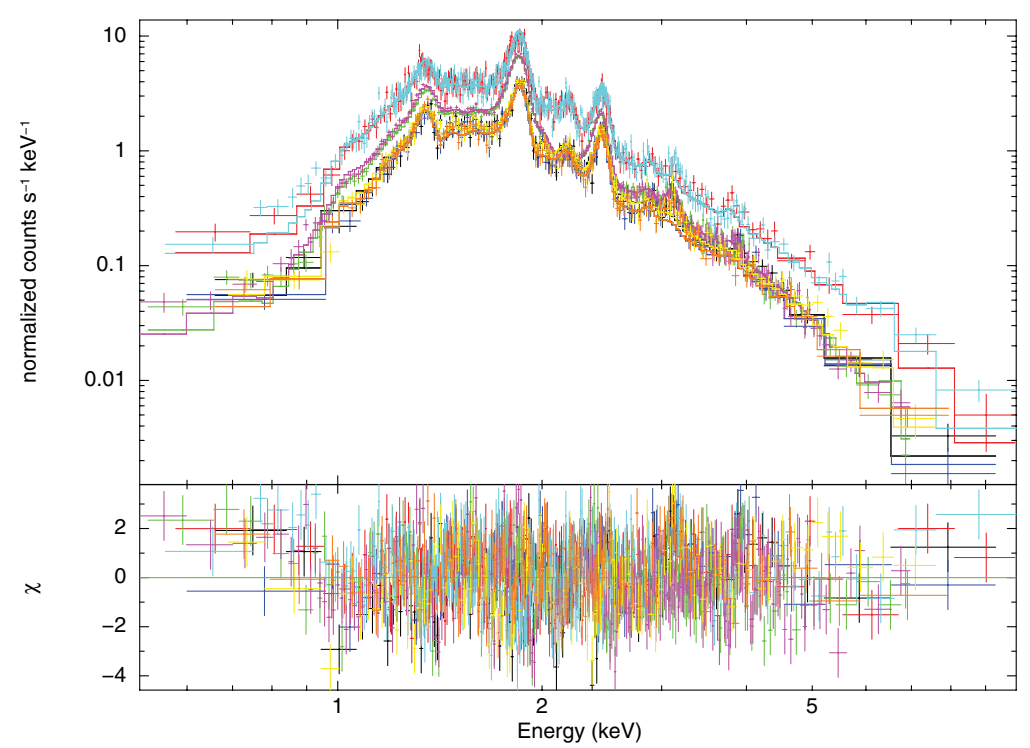

Figure 3. Best-fit VSEDOV+VPSHOCK model to the global SNR spectra using Chandra and XMM-Newton data [pn: red and cyan (top spectra), Chandra: pink and green (middle spectra), and MOS1+2: blue, yellow, orange, and black (bottom spectra)].

and $\sim 2.1 D_{8.5} \mathrm{kyr}$ assuming a Sedov phase of evolution. Under the assumption of an explosion in a uniform ambient medium, the Sedov phase yields a shock velocity of $\sim 1000 \mathrm{~km} \mathrm{~s}^{-1}$, an average ambient density of $\sim 0.5 f^{-1 / 2} \mathrm{~cm}^{-3}$, and an explosion energy of $\sim 2.0 \times 10^{50} f^{-1 / 2}$ ergs (where $f$ is the volume filling factor of the hot component). We compare the derived metal abundances from our spectral fits to the core-collapse nucleosynthesis model yields (e.g., Woosley \& Weaver 1995) and infer a progenitor mass of $\sim(25-30) M_{\odot}$, supporting the earlier prediction of a SN type IIL/b star for Kes 73 (Chevalier 2005). This estimated progenitor mass is comparable to that determined for a few other magnetars and high-magnetic field radio pulsars (Safi-Harb \& Kumar 2012), suggesting very massive progenitors for the majority of highly magnetized neutron stars.

This research made use of NASA's ADS and HEASARC. S. Safi-Harb acknowledges support from the CRC program, NSERC, CFI, and CSA.

\section{References}

Chevalier, R. G. 2005, ApJ, 619, 839

Pinheiro Gonçalves, D., Noriega-Crespo, A., Paladini, R., Martin, P. G., \& Carey, S. J. 2011, $A J, 142,47$

Gotthelf, E. V. \& Vasisht, G. 1997, ApJ, 486, L133

Kriss, G. A., Becker, R. H., Helfand, D. J., \& Canizares, C. J. 1985, Apj, 288, 703

Kumar, H. S., Safi-Harb, S., Slane, P. O., \& Gotthelf, E. V. 2013, ApJ, submitted

Lopez, L. A., Ramirez-Ruiz, E., Huppenkothen, D., Badenes, C., \& Pooley, D. A. 2011, ApJ, 732,114

Safi-Harb, S. \& Kumar, H. S. 2012, Proceedings of IAUS 291, Cambridge University Press, Vol 291, 480 (arXiv:1211.5261)

Vasisht, G. \& Gotthelf, E. V. 1997, ApJ, 486, L129

Vink, J. \& Kuiper, L. 2006, MNRAS, 370, 1, L14

Woosley, S. E. \& Weaver, T. A. 1995, ApJSS, 101, 181 